PROCEEDINGS OF THE

AMERICAN MATHEMATICAL SOCIETY

Volume 97. Number 2. June 1986

\title{
NON-ARTINIAN ESSENTIAL EXTENSIONS OF SIMPLE MODULES
}

\author{
K. R. GOODEARL ${ }^{1}$ AND A. H. SCHOFIELD
}

\begin{abstract}
A noetherian ring of Krull dimension one is constructed which possesses a simple module with a cyclic non-artinian essential extension. The construction also provides an example of a torsionfree noetherian bimodule which fails to satisfy Jategaonkar's density condition.
\end{abstract}

A crucial step in Jategaonkar's proof of Jacobson's conjecture for fully bounded noetherian rings is that over such rings, any finitely generated essential extension of an artinian module is artinian [3, Corollary 3.6; 1, Theorem 7.10]. The natural question, whether this holds over arbitrary noetherian rings [3, p. 116], was answered in the negative by Musson [6, Theorems 4.1, 4.2; 7, Theorem 1; 1, Example 7.15]. Musson constructed counterexamples over prime noetherian rings of Krull dimensions $2,3,4, \ldots$, but the question remained open in Krull dimension one [1, Remark 4, p. 108]. Also, since Lenagan proved Jacobson's conjecture for noetherian rings of Krull dimension one [5, Theorem 4.4; 1, Theorem 5.13], it was natural to ask whether this case of Jacobson's conjecture holds because finitely generated essential extensions of artinian modules are artinian.

Over a semiprime noetherian ring of Krull dimension one, all factors of the ring by essential one-sided ideals are artinian [2, Proposition 6.1], whence all factors of finitely generated modules by essential submodules are artinian. In particular, all finitely generated essential extensions of artinian modules are artinian. Thus, for this condition to fail over a noetherian ring of Krull dimension one, the prime radical must be nonzero.

Our construction, which takes the form of a triangular matrix $\operatorname{ring}\left(\begin{array}{cc}R & 0 \\ R & S\end{array}\right)$, requires an extension $R \supset S$ of noetherian rings of Krull dimension one such that ${ }_{S} R$ is finitely generated and $R$ has a maximal right ideal $M$ for which $S \cap M=0$. We shall obtain $R$ and $S$ as polynomial rings $E[t]$ and $F[t]$ for a suitable extension $E \supset F$ of skew fields. For ${ }_{S} R$ to be finitely generated, we need only that ${ }_{F} E$ be finite-dimensional. Our maximal right ideal $M$ will be of the form $(t-\alpha) E[t]$, where $\alpha$ is transcendental in the following sense.

Received by the editors April 29, 1985.

1980 Mathematics Subject Classification. Primary 16A33.

Key words and phrases. Noetherian ring, simple module, essential extension.

${ }^{1}$ The research of the first author was partially supported by a grant from the U.S. National Science Foundation.

(c)1986 American Mathematical Society $0002-9939 / 86 \$ 1.00+\$ .25$ per page 
Definition. Let $E \supset F$ be skew fields. An element $\alpha \in E$ is right transcendental over $F$ provided the powers of $\alpha$, namely $1, \alpha, \alpha^{2}, \ldots$, are right linearly independent over $F$. The extension $E \supset F$ is right transcendental if and only if $E$ contains an element right transcendental over $F$.

LEMMA 1. Let $E \supset F$ be skew fields, let $\alpha \in E$, and let $t$ be a central indeterminate. If $\alpha$ is right transcendental over $F$, then

$$
F[t] \cap(t-\alpha) E[t]=0 .
$$

Proof. Consider any polynomial

$$
p=\beta_{0}+\beta_{1} t+\cdots+\beta_{n} t^{n} \in F[t] \cap(t-\alpha) E[t] .
$$

Then there is a polynomial $\gamma_{0}+\gamma_{1} t+\cdots+\gamma_{n-1} t^{n-1}$ in $E[t]$ such that

$$
\beta_{0}+\beta_{1} t+\cdots+\beta_{n} t^{n}=(t-\alpha)\left(\gamma_{0}+\gamma_{1} t+\cdots+\gamma_{n-1} t^{n-1}\right) .
$$

Then $\beta_{0}=-\alpha \gamma_{0}$ and $\beta_{n}=\gamma_{n-1}$, while $\beta_{i}=\gamma_{i-1}-\alpha \gamma_{i}$ for $i=1, \ldots, n-1$. By induction,

$$
\gamma_{i}=\beta_{i+1}+\alpha \beta_{i+2}+\cdots+\alpha^{n-1-i} \beta_{n}
$$

for $i=n-1, n-2, \ldots, 0$. Consequently,

$$
\beta_{0}+\alpha \beta_{1}+\cdots+\alpha^{n} \beta_{n}=\beta_{0}+\alpha \gamma_{0}=0
$$

As $\alpha$ is right transcendental over $F$, all the $\beta_{i}=0$, whence $p=0$.

Now our task is to construct a skew field extension which is left finite-dimensional and right transcendental. To this end, it is convenient to quote [8, Theorem 5] in the following form.

THEOREM 2. Let $E \supset F$ be an extension of skew fields, and let $e_{1}, \ldots, e_{n}$ be elements of $E$ left linearly independent over $F$. If $n \geqslant 2$, there exist skew fields

$$
\begin{aligned}
& E^{\prime} \supset F^{\prime} \\
& \cup \quad \cup \\
& E \supset F
\end{aligned}
$$

such that $\left\{e_{1}, \ldots, e_{n}\right\}$ is a left basis for $F^{\prime} E$ over $F^{\prime}$ while all subsets of $E$ which are right linearly independent over $F$ remain right linearly independent over $F^{\prime}$.

THEOREM 3. Let $E_{0} \supset F_{0}$ be an extension of skew fields, and let $e_{1}, \ldots, e_{n}$ be elements of $E_{0}$ left linearly independent over $F_{0}$. If $n \geqslant 2$, there exist skew fields

$$
\begin{aligned}
& E \supset F \\
& \cup \cup \cup \\
& E_{0} \supset F_{0}
\end{aligned}
$$

such that $\left\{e_{1}, \ldots, e_{n}\right\}$ is a left basis for $E$ over $F$ while all subsets of $E_{0}$ which are right linearly independent over $F_{0}$ remain right linearly independent over $F$. 
Proof. By induction, we construct a tower of skew field extensions

$$
\begin{array}{cc}
\vdots & \vdots \\
\cup & \cup \\
E_{1} \supset F_{1} \\
\cup & \cup \\
E_{0} \supset F_{0}
\end{array}
$$

such that $\left\{e_{1}, \ldots, e_{n}\right\}$ is a left basis for each $F_{j+1} E_{j}$ over $F_{j+1}$, while all subsets of $E_{j}$ which are right linearly independent over $F_{j}$ remain right linearly independent over $F_{j+1}$. If the tower has been constructed as far as the extension $E_{m} \supset F_{m}$, then, in particular, the elements $e_{1}, \ldots, e_{n}$ are left linearly independent over $F_{m}$ (since if $m>0$ they form a basis for $F_{m} E_{m-1}$ over $F_{m}$ ). Then Theorem 2 shows that there exist skew fields

$$
\begin{array}{lll}
E_{m+1} & \supset F_{m+1} \\
\cup & \cup \\
E_{m} \supset F_{m}
\end{array}
$$

such that $\left\{e_{1}, \ldots, e_{n}\right\}$ is a left basis for $F_{m+1} E_{m}$ over $F_{m+1}$ while all subsets of $E_{m}$ which are right linearly independent over $F_{m}$ remain right linearly independent over $F_{m+1}$. This completes the inductive part of the construction.

Let $E=\bigcup E_{j}$ and $F=\bigcup F_{j}$. Each element of $E$ lies in some $E_{j}$ (and hence in $\left.F_{j+1} E_{j}\right)$ and must therefore lie in the left span of $\left\{e_{1}, \ldots, e_{n}\right\}$ over $F_{j+1}$ and also over $F$. Moreover, since the elements $e_{1}, \ldots, e_{n}$ are left linearly independent over each $F_{j+1}$, they are left linearly independent over $F$. Therefore, $\left\{e_{1}, \ldots, e_{n}\right\}$ is a left basis for $E$ over $F$. Further, given any subset $X \subset E_{0}$ that is right linearly independent over $F_{0}$, our construction ensures that $X$ remains right linearly independent over each $F_{j+1}$, and therefore right linearly independent over $F$.

COROllaRY 4. Given any integer $n \geqslant 2$, there exist skew field extensions $E \supset F$ such that on the left, $E$ is $n$-dimensional over $F$, while on the right, $E$ is transcendental over $F$.

Proof. We take $F_{0}$ to be a commutative field $K$, and $E_{0}$ to be a rational function field $K(x)$. Then $1, x, x^{2}, \ldots, x^{n}, \ldots$ are (left and right) linearly independent over $F_{0}$. When we apply Theorem 3 , choosing $x, x^{2}, \ldots, x^{n}$ for $e_{1}, \ldots, e_{n}$, we obtain skew fields

$$
\begin{aligned}
& E \supset F \\
& \cup \quad \cup \\
& E_{0} \supset F_{0}
\end{aligned}
$$

such that $\left\{x, x^{2}, \ldots, x^{n}\right\}$ is a left basis for $E$ over $F$ while $\left\{1, x, x^{2}, \ldots\right\}$ is right linearly independent over $F$.

EXAMPLE. There exists a right and left noetherian ring with right and left Krull dimension one which has a simple module with a non-artinian cyclic essential extension.

Proof. By Corollary 4, there exists a skew field extension $E \supset F$ such that $E$ is left finite-dimensional over $F$ while $E$ contains an element $\alpha$ which is right 
transcendental over $F$. Set $R=E[t]$ and $S=F[t]$, where $t$ is a central indeterminate, and set $T=\left(\begin{array}{cc}R & 0 \\ R & S\end{array}\right)$. Since ${ }_{F} E$ is finite-dimensional, ${ }_{S} R$ is finitely generated, whence $T$ is right and left noetherian. The ideal $N=\left(\begin{array}{cc}0 & 0 \\ R & 0\end{array}\right)$ is the prime radical of $T$, and $T / N \cong R \times S$. Thus $T / N$ has right and left Krull dimension one, and (since $N^{2}=0$ ) the same is clearly true of $T$.

Set $M=(t-\alpha) R$, which is a maximal right ideal of $R$. Since $\alpha$ is right transcendental over $F$, Lemma 1 shows that $S \cap M=0$. Set

$$
I=\left(\begin{array}{cc}
0 & 0 \\
M & 0
\end{array}\right), \quad J=\left(\begin{array}{cc}
0 & 0 \\
R & 0
\end{array}\right), \quad K=\left(\begin{array}{cc}
0 & 0 \\
R & S
\end{array}\right),
$$

which are right ideals of $T$. Then $J / I$ is a simple right $T$-submodule of the cyclic right $T$-module $K / I$. Since $S$ is not right artinian, $K / J$ is a non-artinian right $T$-module, whence $K / I$ is non-artinian.

It remains to be shown that $J / I$ is essential in $K / I$. Any nonzero element of $K / I$ is a coset $x+I$, where $x \in K-I$. If $x \in J$, then $x+I$ is in $J / I$. If $x \notin J$, then $x=\left(\begin{array}{ll}0 & 0 \\ a & b\end{array}\right)$ for some $a \in R$ and some nonzero $b \in S$. As $S \cap M=0$, we have $b \notin M$, whence the product $x\left(\begin{array}{ll}0 & 0 \\ 1 & 0\end{array}\right)=\left(\begin{array}{ll}0 & 0 \\ b & 0\end{array}\right)$ lies in $J-I$, and so $(x+I)\left(\begin{array}{ll}0 & 0 \\ 1 & 0\end{array}\right)$ is a nonzero element of $J / I$. Therefore $K / I$ is an essential extension of $J / I$.

The ingredients in our example also provide a bimodule which fails to satisfy the "right density condition" introduced by Jategaonkar in [4, pp. 53, 54]. (A torsionfree noetherian bimodule ${ }_{S} B_{R}$ over prime noetherian rings $R$ and $S$ satisfies this condition provided every essential right $R$-submodule of $B$ contains a submodule of the form $c B$ where $c$ is a regular element of $S$.) In our example, the ring $R$ is a torsionfree noetherian $(S, R)$-bimodule, and $M$ is an essential right $R$-submodule of $R$, but $c R \nsubseteq M$ for all nonzero elements $c \in S$, because $S \cap M=0$. Thus, ${ }_{S} R_{R}$ fails to satisfy the right density condition. In fact, the existence of torsionfree noetherian bimodules for which the density condition fails follows from the existence of a noetherian ring of Krull dimension one having a non-artinian finitely generated module with essential socle [4, Corollary 3.6].

\section{REFERENCES}

1. A. W. Chatters and C. R. Hajarnavis, Rings with chain conditions, Pitman, London, 1980.

2. R. Gordon and J. C. Robson, Krull dimension, Mem. Amer. Math. Soc. no. 133 (1973).

3. A. V. Jategaonkar, Jacobson's conjecture and modules over fully bounded noetherian rings, J. Algebra 30 (1974), 103-121.

4. Solvable Lie algebras, polycyclic-by-finite groups, and bimodule Krull dimension, Comm. Algebra 10 (1982), 19-69.

5. T. H. Lenagan, Noetherian rings with Krull dimension one, J. London Math. Soc. (2) 15 (1977), 41-47.

6. I. M. Musson, Injective modules for group rings of polycyclic groups. II, Quart. J. Math. Oxford Ser. (2) 31 (1980), 449-466.

7. Some examples of modules over noetherian rings, Glasgow Math. J. 23 (1982), 9-13.

8. A. H. Schofield, Artin's problem for skew field extensions, Math. Proc. Cambridge Philos. Soc. 97 (1985), 1-6.

Department of Mathematics, University of Utah, Salt lake City, Utah 84112 (Current address of K. R. Goodearl)

\section{Trinity College, Cambridge CB2 1TQ, England}

Current address (A. H. Schofield): Department of Mathematics, University College London, Gower Street, London, WC1E 6BT, England 